\title{
Reflets
}

Revue d'intervention sociale et communautaire

\section{Transformations contemporaines de la paternité : la fin du patriarcat?}

\section{Denyse Côté}

Volume 15, numéro 1, 2009

Paternités : enjeux et perspectives (Première partie)

URI : https://id.erudit.org/iderudit/029587ar

DOI : https://doi.org/10.7202/029587ar

Aller au sommaire du numéro

Éditeur(s)

Reflets : Revue d'intervention sociale et communautaire

ISSN

1203-4576 (imprimé)

1712-8498 (numérique)

Découvrir la revue

Citer cet article

Côté, D. (2009). Transformations contemporaines de la paternité : la fin du patriarcat? Reflets, 15(1), 60-78. https://doi.org/10.7202/029587ar

\section{Résumé de l'article}

Le modèle du père seul responsable de l'autorité, de la discipline et du pourvoi au sein de sa famille est en voie de disparition et de remplacement par celui du " père soignant ». La nouvelle fluidité des rôles parentaux, la mobilité accrue des mères (entre la famille et le marché du travail, entre conjoints et ex-conjoints, entre famille intacte et recomposée) auraient-elles sonné le glas de la famille patriarcale en Occident? Le patriarcat est-il désormais relégué aux oubliettes de l'histoire? Cet article propose une analyse sociologique de la paternité et de son évolution récente en regard du concept du patriarcat dans le contexte des sociétés occidentales contemporaines.
Tous droits réservés @ $@$ Reflets : revue d'intervention sociale et communautaire, 2009
Cocument est protégé par la loi sur le droit d'auteur. L'utilisation des services d’Érudit (y compris la reproduction) est assujettie à sa politique d'utilisation que vous pouvez consulter en ligne.

https://apropos.erudit.org/fr/usagers/politique-dutilisation/ 


\section{Transformations contemporaines de la paternité : la fin du patriarcat?}

\section{Denyse Côté,}

Professeure titulaire, Directrice de l'Observatoire sur le développement régional et l'analyse différenciée selon les sexes (ORÉGAND)

Cet article a pour objectif de mieux comprendre les concepts de paternité et de patriarcat ainsi que leurs mutations contemporaines. La prétention n'est pas d'articuler les différentes théories du patriarcat ou des rôles parentaux, mais plutôt d'articuler la réalité empirique et les représentations changeantes de la paternité avec le concept de patriarcat. Nos analyses sont ancrées en sociologie de la famille et des rapports sociaux de sexes; elles s'inspirent de plusieurs recherches empiriques sur la transformation des rôles parentaux (Côté, 2006, 2004, 2002, 2000).

Le sujet abordé dans cet article en est un de discussion courante et fait l'objet de nombreuses interventions et politiques publiques. Il s'agit aussi d'un sujet hautement normatif; en effet, la nécessité d'une famille fonctionnelle pour le sain développement d'un enfant est au coeur de différents discours sociaux, religieux et scientifiques et des croyances profondes de la majorité d'entre nous.

Mais quelle devrait être la configuration de cette famille? Il y a déjà presque quarante ans, des mouvements sociaux, en particulier le mouvement féministe, ont appelé au décloisonnement des rôles parentaux, les femmes étant jusque-là les seules à assumer la charge éducative quotidienne des enfants et obligées de leur consacrer leurs vies. Plus récemment, le mouvement des pères a appelé à la reconnaissance de la capacité de ces derniers à prendre en charge le quotidien de leurs enfants après la séparation ou le divorce. 
Les théories psychologiques ont, depuis le milieu du siècle dernier, ségrégé les rôles sexuels et ont campé des formes familiales hétérosexuelles comme "normales " et nécessaires. La sociologie de la famille a à cette époque été dominée par les thèses structurofonctionnalistes (Parsons et Bales, 1955) et encore aujourd'hui, la fluidité émergente des rôles parentaux est contestée par les approches dominantes en Amérique du Nord et en Europe.

Les régulations sociales, politiques et morales dont la famille a été l'objet, à toutes les époques et dans toutes les sociétés, se sont opérées à travers divers discours et dispositifs : lois, pratiques judiciaires, politiques publiques, configurations institutionnelles. Ce sont les discours scientifiques qui fondent les discours politiques, populaires et moraux régulateurs de l'activité domestique au sein des sociétés occidentales contemporaines. Ceux-ci sont à leur tour souvent présentés comme "objectifs "; dans les faits, ils sont construits à partir de projets de recherche circonstanciés, limités et relatifs. Il est ainsi souvent étonnant de constater à quel point de nombreuses recherches sur l'activité domestique ont conservé de tels a priori moraux ou naturalistes fondant la division sexuelle des rôles parentaux ou encore, sont construites à partir de représentations personnelles ou de croyances plutôt que de faits scientifiquement démontrés.

\section{Comment appréhender la paternité comme phénomène social?}

Pour appréhender correctement la paternité, il faut à notre avis distinguer d'entrée de jeu les discours des pratiques de prise en charge des enfants au sein de familles. Car la paternité, comme tout phénomène social, est le produit tant de pratiques que de discours et doit être analysée dans le contexte de procédures et d'institutions sociales qui l'encadrent. En plus du processus identitaire qui lui est propre, la paternité est une relation affective et sociale ancrée, mais dans un environnement donné. Il ne s'agit 
"Car la paternité, comme tout

phénomène social, est le produit tant de pratiques que de discours et doit être analysée dans le contexte de procédures et d'institutions sociales qui l'encadrent. En plus du processus identitaire qui lui est propre, la paternité est une relation affective et sociale ancrée, mais dans un environnement donné.» pas seulement d'un rôle, mais aussi d'un rapport social qui, comme tous les rapports sociaux, se construit et est appelé à se transformer. Pour saisir ce rapport social, nous avons fait appel aux théories du constructivisme (Berger et Luckman, 1989) et de l'actionnalisme (Giddens, 1995).

Celles-ci nous permettent de poser les actions des individus comme conditionnées par une série de contraintes structurelles et de rapports à des " objets pratiques " qui leur donnent un sens (Young, 1994, 1997).Ainsi, la prise en charge du soin des enfants, le fait d'avoir des projets (avoir et éduquer des enfants) et de les réaliser, construirait les identités et les expériences des personnes. Ces actions sont situées dans le cadre d'une existence contrainte par des structures, mais non déterminée. Jusqu'à présent, le rapport des femmes à ces actions et à ces contraintes a transformé les femmes en mères et les hommes en pères. Jusqu'à récemment, les femmes étaient posées comme féminines par une série d'activités articulées autour de structures familiales précises et de " devoirs " maternels. Les hommes étaient posés comme masculins par leur rapport à la sphère publique, au pourvoi et à l'autorité au sein de la famille (Young, 1994, 1997). Pour bien saisir la paternité, il faut donc poser le rapport des hommes et des femmes aux soins des enfants comme constitutif des formes historiquement et culturellement déterminées de parentalité.

Les actrices et acteurs sociaux (les mères et les pères) donnent ainsi continuellement un sens à leurs actions qu'ils insèrent dans le cadre de stratégies personnelles. Ils se définissent personnellement et socialement en rapport avec des objets (le soin et l'éducation de leurs enfants) et des structures et sont actifs dans la production de la société (Walby, 1990). Leurs actions créent dans une certaine mesure les histoires qu'ils ou elles vivent. Si l'individu est le produit d'une société, à leur tour, les individus contribuent en permanence à produire cette société. L'histoire serait alors la conséquence des actions stratégiques et des discours de ces différents acteurs ou actrices ainsi que des effets non intentionnels de leurs actions. Cela serait vrai pour les pères et les mères qui, loin de reproduire automatiquement des comportements appris, créeraient plutôt dans leur vie quotidienne des habitus, ou systèmes de dispositions 
durables et transposables qui intègrent des expériences passées et fonctionnent à chaque moment comme des matrices de perceptions, d'appréciations et d'actions (Bourdieu, 2000). De tels habitus doivent être mis en rapport avec les structures sociales qui définissent leurs conditions de reproduction.

Ainsi, l'attribution sexuée du soin des enfants a,jusqu'à présent, construit la paternité et la maternité, elle a construit le genre (Devault, 1994). Car, le soin des jeunes enfants a habituellement été attribué aux mères qui l'ont partagé ou confié à d'autres femmes, qu'elles soient gardiennes, gouvernantes, éducatrices, jeunes filles au pair, grands-mères, tantes ou filles. Mais il est rare que le père ait pris en charge ces tâches, en particulier lorsque les mères étaient présentes et actives auprès d'eux. On leur a relégué une fonction symbolique importante, un rôle d'autorité et de pourvoi.

Analyser la prise en charge parentale des enfants, c'est donc se pencher sur un phénomène qui est à la fois matériel et non matériel (Juteau-Lee, 1983), les discours et les pratiques devant tous deux faire l'objet d'analyses. Car l'aspect matériel est intrinsèquement lié à la production de sens. Il faut prendre en compte les actions, aussi bien que la force et la prégnance des représentations sociales en matière de vie domestique. Car, les représentations dominantes ont été un élément régulateur important des pratiques domestiques (Donzelot, 2005) et un frein à leur appréhension sociologique.

Nous avons dans nos recherches empiriques (Côté, 2000, 2006) appréhendé la paternité à travers les pratiques de soin aux enfants. Cela offre l'avantage d'éliminer le poids d'objets sociologiques construits à partir d'a priori et de discours scientifiques qui reflètent l'attribution nécessaire et automatique du pourvoi et de l'autorité aux hommes. Cela nous a permis d'analyser la paternité non pas comme une attribution naturelle, mais comme une logique d'organisation du social qui forme un système ou même, selon certains auteurs, une institution sociale (Delphy, 1974). Nous avons abordé ce phénomène à travers le concept de travail de soin, car celui-ci nous permettait de saisir l'activité productrice d'énergies humaines. Il s'agit en effet de l'activité qui crée et fonde le rapport parental et comporte des dimensions matérielles, relationnelles, émotives, éducatives et sociales. Ce 
travail nécessaire au soin et à l'éducation des enfants réfere à l'activité (le soin) plutôt qu'au cadre dans lequel elle s'effectue (la famille), au rôle de la personne qui en a eu généralement la charge (la mère et maintenant, de plus en plus, le père) ou au sentiment qui en constitue le cadre normatif (l'amour). Parler de soin aux enfants, c'est référer à l'ensemble des travaux d'entretien matériel et affectif, à l'ensemble des interventions éducatives ainsi qu'aux considérations organisationnelles, économiques, psychologiques dont une personne adulte se charge sur une base quotidienne et régulière pour les enfants dont elle est responsable. Ce travail produit la socialisation des nouveaux-nés qui, à son tour, produit des êtres humains adultes (Juteau-Lee, 1983).

\section{La paternité : du symbole à la contingence}

La paternité et la maternité se sont en fait construites de façon parallèle et complémentaire sur le fait que les parents prennent soin des enfants pour une période prolongée, sur la base d'une attribution sexuée du soin aux enfants et, dans la plupart des cas, dans le cadre du partage d'une vie domestique entre conjoints de sexe différent. Pour mieux saisir par la suite l'exercice de la paternité dans la société contemporaine, un retour s'impose sur son évolution et son exercice.

Les discours sur les pères ont jusqu'à tout récemment été fondés sur la contingence et l'immatérialité (Griswold, 1994; Ehrenreich et English, 1989). Le père symbolique conserve une place importante dans le processus de filiation (Tahon, 2004) et le père psychique semble avoir remplacé le père spirituel d'antan, parrain, confesseur ou directeur de conscience. La version lacanienne de ce père psychique fait appel à une loi intérieure du sujet qui structurerait les rapports de l'individu face à lui-même et au monde extérieur. Cette dimension symbolique s'entrecroise avec les dimensions sociale et légale au sein d'anciens discours ou de discours en émergence. Ainsi, l'absence du père est encore 
souvent associée dans les discours professionnels et scientifiques à des comportements individuels déviants, provoquant chez l'enfant un mal d'identification trop souvent associé automatiquement à la déviance (Knibiehler, 1997).

De telles explications psychanalytiques entrecroisent souvent certaines explications naturalistes. Dans une version très répandue, le père serait l'intermédiaire entre la nature (dont la femme serait plus proche) et la culture, définie comme immatérielle. Il s'interposerait dans une relation mère-enfant dyadique, charnelle et sensuelle. Il exercerait ainsi une fonction de séparation, de régulation de la distance mère-enfant et susciterait ainsi l'émergence de la personnalité de l'enfant (Chaudey, 1990); il interviendrait sur le plan de son développement psychoaffectif et cognitif. La relation au père serait alors triadique et abstraite; elle introduirait au monde de la pensée, du langage, de l'abstraction, de la logique et de la rationalité. La présence du père serait nécessaire, mais contingente dans le temps, c'est-à-dire qu'elle n'entraînerait pas l'obligation d'une présence continue.

Une autre thèse associe l'explication psychologique à l'explication sociologique et pose le père comme essentiel à la formation de l'identité sexuelle des garçons. Par exemple, la fonction de socialisation du père chez Parsons et Bales (1955) réfere à la production d'enfants normaux qui intérioriseraient par voie de modélisation parentale différenciée des rôles sexuels adéquats. Les pères devraient pour cela refouler tout comportement relié au maternage des enfants et développer plutôt les attitudes et comportements nécessaires au maintien et à la défense d'un statut familial par le biais du pourvoi. La présence régulière du père serait donc ici très importante, mais nécessairement articulée à son rôle instrumental. L'absence physique continue du père aurait une répercussion négative sur les garçons, mais paradoxalement, sa présence, toujours modulée aux exigences du rôle paternel, serait en toutes circonstances positive. Elle servirait aussi de frein à la délinquance, et même de rempart contre l'homosexualité. Cette idée est encore très populaire aujourd'hui, malgré l'absence de preuves pouvant la fonder, car les recherches sur la formation d'une identité sexuelle chez les garçons élevés sans leur père 
démontrent que ceux-ci sont parfois moins masculins, parfois au contraire hypermasculins (Griswold, 1994).

Cette tradition psychanalytique confere au père une place plus symbolique que matérielle, ce qui a obligé beaucoup de mères à jouer un rôle central dans le modelage des identités tant masculine que féminine (Chodorow, 1999) et a conféré au père la symbolique de l'autorité plutôt que celle d'un père inscrit dans le corps (Olivier, 1994). L'enfant s'attacherait et voudrait en fait être aimé de celui ou de celle qui s'en occupe et cette personne serait inscrite dans son psyché.

\section{La paternité : une construction sociale de l'autorité}

Cette construction symbolique du père référait jusqu'à tout récemment à une autorité paternelle consignée juridiquement, remontant au temps des Romains, réaffirmée en Europe lors de la Renaissance et de la montée de l'absolutisme, et conjuguée à un ordre religieux. D'ailleurs, patriarcaux de nature, tous les mouvements fondamentalistes, catholiques, musulmans ou protestants, ont posé ou posent le père comme garant de l'ordre et de la discipline. Cette image de l'autorité paternelle réfere dans la tradition juridique québécoise au droit romain et au Code napoléonien, ancêtres du Code civil. En retour de son autorité, qui n'était pas absolue, mais bien limitée par certains facteurs ${ }^{1}$, le père devait protection et pourvoi aux membres de sa famille. La Révolution française a promulgué les premières lois limitant la puissance paternelle (Knibiehler, 1997) et le libéralisme a par la suite séparé le pouvoir paternel du pouvoir politique, reflétant ainsi cette séparation croissante entre les sphères privée et publique. La prépondérance du père recula au XIX ${ }^{\mathrm{e}}$ siècle au profit de trois forces nouvelles : l'État, la mère et la science (Knibiehler, 1997). Néanmoins, ce n'est que tout récemment que les dernières traces légales de l'autorité paternelle ont disparu :la correction paternelle a disparu en France en 1935, les femmes mariées ont récupéré le statut d'adulte au Québec en 1963 et l'autorité parentale conjointe 
"Selon certains, les pères canadiensfrançais auraient été privés du privilège de cette autorité paternelle, car ils n'auraient occupé qu'une place au bas de l'échelle de la sphère publique, étant issus d'un groupe colonisé qui n'avait accès qu'à des emplois peu intéressants et peu lucratifs. » s'est substituée à l'autorité paternelle au Québec en 1980 et en France en 2002.

Selon certains, les pères canadiens-français auraient été privés du privilège de cette autorité paternelle, car ils n'auraient occupé qu'une place au bas de l'échelle de la sphère publique, étant issus d'un groupe colonisé qui n'avait accès qu'à des emplois peu intéressants et peu lucratifs. En conséquence, les pères canadiensfrançais se seraient réfugiés dans l'absence et dans le silence. Mais l'autorité paternelle a bel et bien été enchâssée dans le Code civil québécois jusqu'à la Révolution tranquille et elle a été exercée sous la tutelle de l'Église catholique. Quelle jouissance les pères québécois en ont-ils eue? Celle que leur permettait leur milieu social, économique, culturel et religieux; mais l'expérience des hommes était certainement teintée de cette symbolique et de ce rôle.

Il semble donc que la vie familiale se présente à l'homme canadien-français comme une activité d'ordre de pouvoir sur les autres. L'homme a l'ascendance dans le groupe familial parce qu'il a l'autorité. La famille comme structure est d'abord l'attribution de l'autorité à l'homme. (Garigue, 1962, p. 34)

Soulignons que le pourvoi et l'autorité paternelle sont intimement reliés. D'ailleurs, la reconnaissance légale de l'autorité dévolue au mari par le droit coutumier anglais entraînait la responsabilité du pourvoi; mais celui-ci pouvait s'effectuer grâce aux revenus ou propriétés personnelles de l'épouse ou des enfants (Bernard, 1983).

Aujourd'hui, le discours clérico-nationaliste canadien-français sur l'autorité paternelle est enterré. Le dernier clou dans le cercueil de l'autorité paternelle et de la discipline comme principes éducatifs (Drakish, 1989) a été planté par la contre-culture des années 1960 et 1970 que d'aucuns ont décrite comme constitutive d'une révolte contre le père (Cooper, 1976). On ne réfêre plus à l'autorité paternelle, sauf lorsqu'on souligne son absence, telle l'absence présumée de discipline paternelle au sein des familles 
monoparentales à chef féminin. Mais on voit toutefois émerger de nouveaux discours sur l'autorité paternelle, issus de la nouvelle droite américaine ou d'autres groupes politico-religieux à tendance conservatrice.

\section{La paternité : une construction sociale du pourvoi}

"Ce rôle du père comme bon pourvoyeur continua à évoluer et cela s'observa en particulier pendant la Grande Dépression, source de profonde remise en question sur ce plan, les coûts psychiques de la perte du pourvoi ayant été énormes pour les hommes, les familles et le système américains..."
Le père n'a pas été qu'un symbole et un dépositaire de l'autorité. La réalité des pères a été multiforme et l'est encore : elle reflète les cultures, classes sociales, situations économiques et politiques de différentes époques et sociétés. Elle a été incarnée, contingente et présente dans la vie quotidienne. Ainsi, entre les $\mathrm{XI}^{\mathrm{e}}$ et $\mathrm{XVI}^{\mathrm{e}}$ siècles en Europe, paternité et transmission du patrimoine allaient de pair chez les aristocrates; par contre, le paysan français n'aura appris à ses fils qu'à se soumettre aux exigences de la terre tandis que le citadin leur aura transmis un métier ou un commerce (Knibiehler, 1997). La ruine des entreprises familiales lors de la Révolution industrielle a mené à la création d'une territorialité sexuelle différenciée : vie familiale et emploi ont été séparés. Les temps d'échange entre hommes et femmes ont diminué et, graduellement, l'identité sexuelle a été associée au lieu et à la nature du travail. Les pères ont ainsi été associés à la vie professionnelle, au détriment d'une vie familiale qui ne pouvait et ne devait plus s'y conjuguer. Leur travail devint invisible pour la famille et, grâce aussi aux thèses rousseauistes, la mère devint l'agente principale de cette dernière, puis la seule agente responsable directement des enfants. Si le pourvoi a depuis toujours fait partie des représentations du rôle paternel et de ses fonctions, les prérogatives du pourvoyeur ont augmenté après la Révolution industrielle et le succès des hommes au travail en vint dès le début du $\mathrm{XX}^{\mathrm{e}}$ siècle à définir la masculinité, les qualités masculines (force, créativité, endurance, astuce, par exemple) et le statut des hommes dans la famille et dans la communauté (Bernard, 1983). Ce rôle du père comme bon pourvoyeur continua à évoluer et cela s'observa en particulier pendant la Grande Dépression, source de profonde 
remise en question sur ce plan, les coûts psychiques de la perte du pourvoi ayant été énormes pour les hommes, les familles et le système américains (Griswold, 1994). Suivit, après la Deuxième Guerre mondiale, un âge d'or du monopole des hommes sur le pourvoi. La capacité des pourvoyeurs de procurer à leurs épouses et enfants un niveau de vie élevé vint en effet symboliser le succès du système économique et politique américain qui rendait possible une telle abondance (Bernard, 1983).

Reste qu'au cours des trois derniers siècles, le pourvoi aura été en Amérique du Nord l'élément unificateur de la vie des pères. Quoique d'importantes exceptions peuvent être recensées, et l'exemple des esclaves noirs est éloquent à cet égard, il a tout de même traversé les barrières de la race, de l'appartenance de classe et de l'identité personnelle (Bernard,1983; Griswold, 1994). Les pères subvenant aux besoins des enfants, les mères devaient donc rester à la maison, prendre en charge le soin des enfants et occuper, le cas échéant, des emplois plus précaires et définis comme féminins. Même si elle demeurait inaccessible aux foyers les moins fortunés, cette division du travail avait un sens pour plusieurs hommes ainsi que pour leurs conjointes. Elle n'était pas cependant sans contraintes pour les hommes. En effet, certains pères se sont sentis écartelés entre les exigences de leur travail et celles des femmes et de la famille. D'autres ont eu de la difficulté à assurer un pourvoi et à réconcilier la norme du pourvoi et celle de la socialisation des enfants. Enfin, d'autres ont connu des tensions reliées à la rigidité et au cloisonnement étanche du rôle de pourvoyeur et de cette construction de la masculinité. On commence à peine à recenser les réactions des hommes face à cette obligation au pourvoi.

La surperformance et le rejet du rôle de pourvoyeur se sont parfois cristallisés en fuite, elle-même parfois érigée en mythe (Ehrenreich, 1983) ${ }^{2}$. L'absence physique et psychique du père est d'ailleurs une des images les plus fortes de la culture occidentale. Mais vers 1980, le salaire unique n'étant plus suffisant pour soutenir une famille de classe moyenne, le rôle paternel de pourvoyeur s'effondra ${ }^{3}$, de même que le monopole masculin sur le pourvoi. La paternité en fut radicalement transformée. Le 
pourvoi ne prévaut plus autant depuis lors dans la construction de l'identité masculine et paternelle et leur sens culturel devient donc instable. La paternité est même parsemée d'ambiguïtés, le pourvoi étant présenté dans plusieurs cas comme une image réductrice de la paternité.

\section{Nouvelles configurations sociales de la paternité}

"La valeur qu'on accorde à la paternité a aussi changé. De façon générale, les hommes affirment retirer plus de satisfaction de la famille que du travail. Mais déjà en 1976, seulement $49 \%$ des pères sondés avaient un préjugé favorable envers la paternité, contre $63 \%$ en $1957^{4}$.»
La redéfinition actuelle de la paternité illustre bien ce phénomène. Nous ne savons plus ce qu'est un père, dira-t-on. On est intrinsèquement père; être père, c'est tout simplement vivre avec l'enfant, quelle que soit la composition de la famille, traditionnelle ou monoparentale, ou être père c'est participer à l'éducation d'un enfant ou de plusieurs, sans définir la portée de cette participation. Certains pères délaissent le rôle de pourvoyeur. Plusieurs rejettent en effet l'organisation sociale antérieure basée sur la différenciation sexuelle stricte des rôles paternel et maternel.

La valeur qu'on accorde à la paternité a aussi changé. De façon générale, les hommes affirment retirer plus de satisfaction de la famille que du travail. Mais déjà en 1976, seulement $49 \%$ des pères sondés avaient un préjugé favorable envers la paternité, contre $63 \%$ en $1957^{4}$. Et $49 \%$ d'entre eux voyaient en 1976 la paternité comme un fardeau, contre 27 \% seulement en 1957. Pourtant, pendant cette même période, et encore plus depuis, la paternité est devenue beaucoup moins obligatoire. Plus d'hommes (et de femmes) ont cherché leur satisfaction personnelle en dehors de la paternité (ou de la maternité) (Ehrenreich, 1987).

C'est que les hommes ne veulent plus nécessairement d'enfants pour assurer leur lignée, transmettre le patrimoine, assurer leurs vieux jours, ou répondre à des impératifs religieux. Ils veulent plutôt vivre l'expérience de la paternité et établir des relations affectives dont ils ont l'assurance qu'elles pourront durer. À la suite de la déstabilisation du rôle paternel, les pères tenteraient de renforcer leur rapport aux enfants (Dulac, 1992; 1990; Dubeau, Clément et Chamberland, 2005; Devault et Gaudet, 2003; Quéniart 
et Imbeault, 2003). En Occident, une nouvelle représentation du père remplace l'image du pourvoyeur et du père détenteur de l'autorité : celle d'un père qui prend soin du nourrisson, aide aux tâches ménagères, établit une relation directe avec l'enfant et s'implique à la garderie ou à l'école. La relation père-enfant étant maintenant dégagée des contraintes traditionnelles de la paternité, un nouveau romantisme peut alors émerger : c'est la dimension affective plutôt que la dimension autoritaire ou éducative qui y est privilégiée, la même d'ailleurs qu'avait privilégiée Rousseau (1995) dès le XVIII ${ }^{\mathrm{e}}$ siècle.

Ces nouvelles représentations participent au réaménagement du projet de société américain.

Psychologists and child development specialists have thus added their support for the new fatherhood in the context of an approving culture.... In a nation perplexed about family values, it is comforting to learn that nurturant, involved fathers will produce happier, smarter, better-adjusted children. (Griswold, 1994, p. 252)

Cette nouvelle image en est une associée à la classe moyenne

The new fatherhood, then, can become a badge of class, a sign that one has the knowledge, time, and inclination to embrace more progressive visions of parenting. (...) Life may be hectic in the middle class and the husband's paycheck insufficient to cover family expenses, but at least these men can take solace in leading private lives of a better sort than blue-collar working stiffs. (Griswold, 1994 , p. 253-254)

et surgit justement au moment où la paternité semble moins fréquente, en particulier pour les jeunes hommes.

At the same time that increased attention is being given to the role of fathering, it seam a likely that men on average, are spending less time in living arrangements 
where there is an opportunity to occupy the role of father. (Griswold, 1994, p. 221)

Car, s'il existe clairement une transformation de la répartition des tâches parentales (Côté, 2006, 2004,2000), et que celle-ci a et aura une portée sur les identités et les institutions, on constate encore une certaine désynchronisation entre ces nouveaux discours et les nouvelles pratiques paternelles. Certains pères adoptent ainsi plus rapidement le discours et la représentation nouvellement valorisée du père soignant, mais sont moins enclins à se responsabiliser à part entière pour les tâches quotidiennes, souvent répétitives et peu valorisantes, auxquelles ils renvoient.

\section{Paternité et société patriarcale}

Le concept de paternité pèse lourdement dans nos représentations sociales, parce qu'il rappelle une époque récemment révolue où elle était exercée dans sa modalité patriarcale. Mais la paternité se transforme rapidement sous l'influence de rapports hommesfemmes changeants. Mais quel lien doit-on établir entre paternité et patriarcat? Plus précisément, l'émergence de cette nouvelle paternité soignante sonne-t-elle le glas du patriarcat?

Pour répondre à cette question, il importe de bien comprendre le concept de patriarcat. Étymologiquement, ce terme provient du grec "pater " (père) et "arche » (dirigeant). Il désigne la forme sociale au sein de laquelle les pères détiennent l'autorité et le pouvoir à travers leurs familles respectives (Weber, 1947). "Les hommes ont été (depuis longtemps et dans la plupart des sociétés) les dirigeants politiques et ont aussi exercé l'autorité au sein du foyer " (Mead, 2003, p. 48). Un patriarche serait donc l'homme qui exerce une telle influence sur sa famille et sur la société. À ne pas confondre cependant avec le concept de patrilinéarité (filiation transmise à travers le père) et de patrilocalité (relocalisation des épouses dans la résidence ou la communauté du mari). 
Comme mode d'organisation sociale et politique, le patriarcat remonte à l'Antiquité, aux sociétés grecque et romaine. Il a souvent été présenté comme une forme avancée de civilisation et d'organisation sociale visant le plus grand bien de tous. Les arguments appuyant cette position et mis de l'avant par de grands philosophes (Aristote, par exemple) se fondaient généralement sur la biologie humaine, auxquels étaient greffés des arguments religieux, éthiques et philosophiques. Souvent pragmatiques, ces argumentaires soulignaient la force physique des hommes et l'autorité masculine nécessaire au bon ordre domestique. Plusieurs systèmes législatifs et religieux ont été créés à partir de ces positions éthiques et philosophiques, retirant par la même occasion aux femmes la possibilité d'exercer une pleine citoyenneté.

John Stuart Mill a été un des premiers hommes à décrire au XIX $^{\mathrm{e}}$ siècle cette organisation sociale comme constituant une forme d'esclavage (Mill, 2006). De nombreux écrits et mouvements ont par la suite décrié cette situation, mais c'est l'essor des théories féministes radicales des années 1970 qui a consacré la définition du concept de patriarcat comme celui d'un système oppressif pour les femmes. Ces théories radicales se sont employées à conceptualiser la domination des hommes sur les femmes (plutôt que la domination des hommes sur d'autres hommes propres aux théories marxistes par exemple). Cette période encore relativement récente nous a laissé en héritage un sens et une personnification du patriarcat comme négatif et rétrograde.

Les écrits philosophiques, sociologiques, politiques et anthropologiques sur le patriarcat sont nombreux. Nous ne présenterons pas ici de typologie de ces théories du patriarcat ni ne tenterons d'expliquer la teneur des importants débats qui ont animé les trente dernières années autour de ce thème. La courte explication suivante permettra cependant de répondre à notre question.

Le courant féministe radical a développé le concept de patriarcat aussi bien à travers ses actions que ses analyses; il l'a défini comme un système de domination des hommes sur les femmes permettant d'expliquer la prévalence des inégalités hommesfemmes ainsi que leur continuité dans l'histoire. Cela a permis 
d'introduire toute une série de questions considérées auparavant comme non pertinentes aux sciences sociales parce que relevant de la vie "privée » et de les relier entre elles comme parties prenantes d'un système de domination masculine : les agressions sexuelles, le travail domestique, par exemple. Rejetées par le courant féministe libéral plus influent auprès des États et des institutions internationales, les théories du patriarcat ont néanmoins eu beaucoup d'influence au sein des sociétés occidentales. Elles ont aussi démontré certaines limites, la tendance à l'essentialisme et au réductionnisme biologique n'étant pas la moindre.

Certaines théories du patriarcat rejettent cependant ce biologisme déterministe qui postule que chaque homme, individuellement, se retrouve intrinsèquement dans une position de domination et que chaque femme se retrouverait dans une position de subordination. Ainsi, le patriarcat peut se concevoir comme un système de relations sociales et politiques doté de structures et de pratiques et, sur le plan mésothéorique, de six institutions centrales : un mode de production domestique, le travail salarié, l'État, la violence masculine, la sexualité et la culture (Walby, 1990). Ces institutions auraient des façons de faire changeantes, elles-mêmes le résultat de pratiques sociales antérieures. Pour ce qui est des rapports intra-familiaux,

(through) patriarchal production relations in the household [...] women's household labour is expropriated by their husbands or cohabitees. The woman may receive her maintenance in exchange for her labour, especially when she is not also engaged in waged labour. Housewives are the producing class, while husbands are the expropriating class. (Walby, 1990, p. 21)

Car, les paradigmes essentialistes ont posé les femmes en victimes passives d'un ordre social patriarcal et réduit par la même occasion les mères à de simples reproductrices d'un ordre établi. Ils ont par la même occasion confondu la reproduction d'un ordre social antérieur (reproduction sociale), la réification de pratiques familiales (division sexuelle rigide au sein d'une famille) et la reproduction humaine, tant biologique que sociale (gestation, 
allaitement, accouchement, soin et éducation des enfants). Ils ont aussi réduit la reproduction humaine au processus biologique (Cooper, 1976). Ainsi, les mères ont été peintes par certaines théoriciennes radicales comme des marionnettes plutôt que des agentes de la reproduction humaine et sociale.

$\mathrm{Au}$ contraire, nous posons que les mères sont des actrices sociales, positionnées de façon propre et disposant de ressources matérielles et non matérielles. À ce titre, elles peuvent reproduire ou transformer les rapports sociaux dans le cadre domestique et dans la sphère publique. "An agent who has no options whatsoever is no longer an agent" (Giddens, 1995, p. 63). Objets de domination ou sujets dominés, les femmes évoluent dans un contexte souvent plus contraignant et limitatif que celui que connaissent les hommes, mais sont parties prenantes des rapports sociaux dans lesquels elles s'engagent. De la même façon, les hommes ou les pères ne sont pas automatiquement des sujets dominants ou des oppresseurs, quoique, comme tout acteur social, ils sont positionnés dans un réseau de relations sociales et d'institutions qui, avec leurs propres actions, créent leur genre.

Tous les acteurs sociaux, les hommes comme les femmes, sont,

"Il en découle que les pères qui, aujourd'hui comme hier, refusent d'utiliser leur place au sein du ménage comme source d'autorité, agiraient de façon à diminuer l'institution patriarcale. Cette condition est nécessaire à l'apparition de familles hétérosexuelles non patriarcales, où la négociation remplace l'autorité comme mode d'organisation." sur le plan macrosocial, engagés dans des luttes de pouvoir pour ces ressources matérielles et non matérielles, le pouvoir étant compris comme la capacité d'assurer un résultat, de faire primer ses intérêts ou sa volonté dans une situation donnée. Ces rapports ne sont pas unilatéraux et il existe pour les plus et les moins puissants une possibilité continue de résistance et de négociation.

Il en découle que les pères qui, aujourd'hui comme hier, refusent d'utiliser leur place au sein du ménage comme source d'autorité, agiraient de façon à diminuer l'institution patriarcale. Cette condition est nécessaire à l'apparition de familles hétérosexuelles non patriarcales, où la négociation remplace l'autorité comme mode d'organisation. Cependant, la paternité ne se limite pas à l'exercice de l'autorité; le partage par les pères du pourvoi et des tâches liées aux soins des jeunes enfants constitue aussi un changement majeur dans la construction sociale contemporaine de la paternité. Il permet l'émergence du concept de famille hétérosexuelle démocratique et non oppressive pour 
"De nouvelles représentations sociales des rôles parentaux, de nouvelles mesures concrètes (congés parentaux par exemple) ont changé le cadre dans lequel sont formées les nouvelles générations. Est-ce garant du futur?" les mères. Mais il ne s'agit pas d'un automatisme, car ce principe de partage symétrique des tâches parentales peut aussi cacher des divisions du travail sexuées inégales ainsi que la domination d'un partenaire sur l'autre (Côté, 2004, 2000).

Le patriarcat est un système social global. Pour qu'il disparaisse, toutes ses structures et institutions sociales et politiques doivent être transformées. Les transformations contemporaines des rôles parentaux ont eu des répercussions importantes sur les plans institutionnel et politique, nos sociétés occidentales ayant démontré leur grande capacité d'adaptation. De nouvelles représentations sociales des rôles parentaux, de nouvelles mesures concrètes (congés parentaux par exemple) ont changé le cadre dans lequel sont formées les nouvelles générations. Est-ce garant du futur? Le nouveau modèle de paternité brise de nombreux atavismes, mais ne permettra pas à lui seul d'effectuer un changement durable au sein de notre propre société et des autres sociétés du globe s'il n'est pas accompagné de changements personnels, institutionnels et politiques dans tous les autres champs sociaux.

\section{Bibliographie}

BER GER, Peter L., et Thomas LUCKMANN (1989). The Social Construction of Reality, New York, Anchor Books.

BERNARD, Jessie (1983). "The Good-Provider Role : Its Rise and Fall ", sous la direction de SKOLNICK, Arlene et Jerome SKOLNICK, Family in Transition, Boston, Little, Brown and Company.

BOURDIEU, Pierre (2000). Esquisse d'une théorie de la pratique, Paris, Seuil.

CHAUDEY, Marie (1990). «Du paterfamilias au pater formidable », La Vie, № 2337, p. 71-74.

CHODOROW, Nancy (1999). The Reproduction of Mothering : Psychoanalysis and the Sociology of Gender, Berkeley, University of California Press.

COOPER, David (1976). Death of the Family, New York, Penguin.

CÔTÉ, Denyse (2006). « D'une pratique contre-culturelle à l'idéal-type : la garde partagée comme phénomène social ", Revue québécoise de psychologie, Vol.27, № 1, p. 13-32.

CÔTÉ, Denyse (2004). «La garde physique des enfants : nouvelles solidarités parentales ou renouveau patriarcal? ", Nouvelles questions féministes, Vol.23, № 3, p. 80-95.

CÔTÉ, Denyse (2002). «Le paradigme de l'égalité entre les sexes et la fragmentation familiale : nouvelles pratiques de solidarité ", Sciences pastorales, Vol.21, № 2, p. 303-312.

CÔTÉ, Denyse (2000). La garde partagée : l'équité en question?, Montréal, Éditions du remueménage. 
DEVAULT, Annie, et Judith GAUDET (2003). « Le soutien aux pères de famille biparentale : l'omniprésence de docteur maman ", Service social,Vol.50, No 1, p. 1-29.

DEVAULT, Marjorie L. (1994). Feeding the Family :The Social Organization of Caring as Gendered Work. Chicago, University of Chicago Press.

DELPHY, Christine (1974). "Mariage et divorce : l'impasse à double face », Les temps modernes, mai 1974, p. 1815-1829.

DONZELOT, Jacques (2005). La police des familles, Paris, Éditions de Minuit.

DRAKISH, Janice (1989). "In Search of the Better Parent :The Social Construction of Ideologies of Fatherhood ", Canadian Journal Women and Law, No 3, p. 69-87.

DUBEAU, Diane, Marie-Ève CLÉMENT et Claire CHAMBERLAND (2005). " Le père, une roue du carrosse familial à ne pas oublier! État des recherches québécoises et canadiennes sur la paternité ", Enfances, Familles, Générations, № 3.

DULAC, Germain (1992). «L'intimité masculine en éveil : le désir d'enfant suite à la rupture d'union », Revue internationale d'action communautaire, Vol.27, No 67.

DULAC, Germain (1990). Penser le masculin. Essai sur la trajectoire des militants de la condition masculine et paternelle, Québec, Presses de l'Université Laval.

EHRENREICH, Barbara, et Deirdre ENGLISH (1989). For Her Own Good, New York, Anchor Press.

EHRENREICH, Barbara (1987). The Hearts of Men :American Dreams and the Flight from Commitment, New York, Anchor.

FRASER, Nancy (2008). Unruly Practices : Power, Discourse, and Gender in Contemporary Social Theory, Minnesota, University of Minnesota Press.

GARIGUE, Philippe (1962). La vie familiale des Canadiens français, Montréal, Presses de l'Université de Montréal.

GIDDENS, Anthony (1995). A Contemporary Critique of Historical Materialism, Stanford, Stanford University Press.

GIDDENS, Anthony (1993). The Transformation of Intimacy : Sexuality, Love, and Eroticism in Modern Societies, Stanford, Stanford University Press.

GRISWOLD, Robert L. (1994). Fatherhood in America : A History, New York, Basic Books.

JUTEAU-LEE, Danielle (1983). «La production de l'ethnicité ou la part réelle de l'idéel ", Sociologie et sociétés, $\mathrm{Vol} . \mathrm{XV}, \mathrm{N}^{\circ}$ 2, p. 39-54.

KNIBIEHLER, Yvonne (1997). Les pères aussi ont une histoire, Paris, Hachette.

MEAD, Margaret (2003), Sex and Temperament in Three Primitive Societies, New York, Harper Collins.

MILL, John Stuart (2006). On Liberty and the Subjection of Women, London, Penguin Books.

OLIVIER, Christiane (1994). Les Fils d'Oreste ou la question du père, Paris, Flammarion.

PARSONS, Talcott, et Robert F. BALES (1955). Family, Socialization and Interaction Process, New York, The Free Press.

QUÉNIART, Anne, et Jean-Sébastien IMBEAULT (2003). « La construction d'espaces d'intimité chez les jeunes pères ", Sociologie et sociétés, Vol.35, No 2, p. 183-201.

ROUSSEAU, Jean-Jacques (1995). L'Émile ou De l'éducation, Paris, Gallimard.

TAHON, Marie-Blanche (2004). Vers l'indifférence des sexes? Union civile et filiation au Québec, Montréal, Boréal. 
WALBY, Sylvia (1990). Theorizing Patriarchy, Oxford, Basil Blackwell.

WALBY, Sylvia (1997). Gender Transformations, London, Routledge.

WEBER, Max (1947). The Theory of Social and Economic Organization, New York, The Free Press.

YOUNG, Iris Marion (1997). Intersecting Voices : Dilemmas of Gender, Political Philosophy, and Policy, Princeton, N.J., Princeton University Press.

YOUNG, Iris Marion (1994). "Gender as Sexuality :Thinking About Women As a Social Collective », Signs, Vol.19, № 3, p. 713-738.

\section{Note}

1 Saint Paul avançait que le géniteur devait même respecter, sous certains aspects, la liberté de son enfant.

2 Ehrenreich parle d'une révolte des hommes des années 1950 contre le travail et l'autorité patriarcale comme facteurs centraux de l'identité masculine. À partir des années 1960, la nouvelle identité masculine a reposé sur le désir, délaissant le sens des responsabilités qui lui avait été central jusqu'alors (Ehrenreich, 1983).

3 Il aura prévalu de 1830 à 1980, année ou le U.S. Census cessa d'identifier automatiquement l'homme comme chef de famille (Bernard, 1983).

4 Il aura prévalu de 1830 à 1980, année ou le U.S. Census cessa d'identifier automatiquement l'homme comme chef de famille (Bernard, 1983). 